Special issue of the 3rd International Conference on Computational and Experimental Science and Engineering (ICCESEN 2016)

\title{
Monte Carlo Calculations for Photon Attenuation Studies on Different Solid Phantom Materials
}

\author{
O. Gundogdu ${ }^{a, *}$ U.A. TARIM ${ }^{b}$ And O. Gurler ${ }^{b}$ \\ ${ }^{a}$ Kocaeli University, Umuttepe Campus, 41380, Kocaeli, Turkey \\ ${ }^{b}$ Physics Department, Faculty of Arts and Sciences, Uludag University, Gorukle Campus, 16059, Bursa, Turkey
}

\begin{abstract}
The linear attenuation coefficients of water and some solid phantom materials, namely of solid water RMI-457, of plastic water, of RW3 solid water, and of Perspex were determined by Monte Carlo calculations, for gammaray photons with energies of $59.5,80.9,140.5,356.5,661.6,1173.2$, and $1332.5 \mathrm{keV}$. The calculated values were compared with the experimental results presented by other researchers and with theoretical values obtained using the XCOM database. Good agreement was observed between the calculations, the experimental, and the theoretical values. The results indicate that the process in Monte Carlo code can be followed to determine the equivalency of other materials at several energies. Three shielding factors such as half-value layer, tenth-value layer, and mean free path were calculated at all considered gamma-ray energies.
\end{abstract}

DOI: 10.12693/APhysPolA.132.1032

PACS/topics: 21.60.Ka, 25.20.Dc, 87.66.Xa, 25.60.Dz, 29.27.Eg

\section{Introduction}

The study of fundamentals of interactions of radiation with materials has become an important research area, given the increasing use of radioactive isotopes in many fields. Data on the attenuation of gamma-rays in matter is required for many scientific, engineering, and medical applications [1]. Many studies have recently been carried out on the attenuation coefficients for a number of materials, such as concrete [2-5], cement [5-8], glass systems $[1,9,10]$, soil samples [11-13] etc. The value of the linear attenuation coefficient depends on the incident photon energy, the atomic number, and the density of the absorber. Given that the linear attenuation coefficient depends on density $\rho$, it is expressed as a mass attenuation coefficient $\mu / \rho$, representing the linear attenuation coefficient per unit mass density of the material $[5,14]$. Half-value layer (HVL), tenth-value layer (TVL), and mean free path (mfp) are the additional parameters that are calculated for gamma-ray shielding. The HVL represents the thickness of an absorber that will reduce the gamma radiation to half, while the TVL represents the thickness of an absorber that will reduce the gamma radiation to a tenth of its original intensity [15]. Mean free path is the average distance that the gammaray travels between interactions.

Solid water equivalent phantoms are used extensively for the dosimetry of photon beams in radiation therapy, radiology, nuclear medicine, and radiation safety. Water is the phantom material of choice for both reference and relative dosimetry measurements in radiation therapy [16].

In this work, the results of the Monte Carlo calculations of linear attenuation coefficients for four different

*corresponding author; e-mail: o.gundogdu@kocaeli.edu.tr solid phantom materials, which have been experimentally studied by Hill et al. [16], and water are reported, and the equivalency of these phantom materials is investigated by simulation. Other important shielding parameters, half-value layer, tenth-value layer and mean free path are also presented for the gamma-ray energies under investigation.

\section{Materials and methods}

The Monte Carlo algorithms can in principle provide the highest accuracy and precision in theoretical modeling of physical interactions in a medium, applied in circumstances which are often unavailable for experimental measurements, such as at the interfaces and in high dose gradients. The simulation of a particle history is carried out in a stochastic process, which makes use of the microscopic description of the fundamental interactions [17]. At each simulation step, the particle can undergo any of a set of interactions, the probabilities of which are determined by the physical properties of interactions, the local material, and the geometry properties. At each step, one particular interaction is selected randomly from the set. This interaction determines changes in particle properties - for instance, the energy, direction, and production of secondary particles. Results of the Monte Carlo simulations are accurate even for heterogeneous media, but may require a high number of particles to simulate, and can therefore be rather slow [17].

In this work, we have written a simple Monte Carlo code to simulate the gamma-rays incident on water and four different solid phantom materials. The following phantoms were studied: Solid Water RMI-457 (RMI Gammex, Middleton, WI, USA), Plastic Water (Computerized Imaging Reference Systems Inc., Norfolk, VA, USA), RW3 Solid Water (PTW Freiburg, Germany), and Perspex, as detailed by Hill et al. [16]. The elemental compositions and the physical densities of water and the four phantom materials used in this study are presented in Table I. 
TABLE I

Elemental composition by relative weight and the physical densities of water and the investigated four phantom materials [16].

\begin{tabular}{|c|c|c|c|c|c|}
\hline \multirow[b]{2}{*}{ Elem. } & \multicolumn{5}{|c|}{ Phantom materials } \\
\hline & $\begin{array}{c}\text { Water } \\
\rho=1.000 \\
\mathrm{~g} \mathrm{~cm}^{-3}\end{array}$ & $\begin{array}{c}\text { RMI-457 } \\
\rho=1.030 \\
\mathrm{~g} \mathrm{~cm}^{-3}\end{array}$ & $\begin{array}{c}\text { Plastic water } \\
\rho=1.013 \\
\mathrm{~g} \mathrm{~cm}^{-3}\end{array}$ & $\begin{array}{c}\text { RW3 } \\
\rho=1.045 \\
\mathrm{~g} \mathrm{~cm}^{-3}\end{array}$ & $\begin{array}{c}\text { Perspex } \\
\rho=1.190 \\
\mathrm{~g} \mathrm{~cm}^{-3}\end{array}$ \\
\hline $\mathrm{H}$ & 0.1119 & 0.0809 & 0.0925 & 0.0759 & 0.0805 \\
\hline $\mathrm{C}$ & - & 0.6722 & 0.6282 & 0.9041 & 0.5998 \\
\hline $\mathrm{N}$ & - & 0.0240 & 0.0100 & - & - \\
\hline $\mathrm{O}$ & 0.8881 & 0.1984 & 0.1794 & 0.0080 & 0.3996 \\
\hline $\mathrm{F}$ & - & - & - & - & - \\
\hline $\mathrm{Cl}$ & - & 0.0013 & 0.0096 & - & - \\
\hline $\mathrm{Ca}$ & - & 0.0232 & 0.0795 & _ & - \\
\hline $\mathrm{Br}$ & - & - & 0.0003 & - & - \\
\hline $\mathrm{Ti}$ & - & - & - & 0.0120 & - \\
\hline
\end{tabular}

For each material, the linear attenuation coefficients $\mu$ at $59.5,80.9,140.5,356.5,661.6,1173.2$ and $1332.5 \mathrm{keV}$ photon energies were determined using Beer-Lambert law, which states that the intensity of the transmitted gamma-rays, $I$, is given by

$$
I=I_{0} \mathrm{e}^{-\mu x},
$$

where $I_{0}$ is the intensity of the incident beam, and $x$ is the thickness of the absorber.

The transmitted photon intensity was determined using a simple algorithm. In this algorithm, a beam of $10^{6}$ incident photons was directed towards the attenuator and was followed, as explained below. The coordinates of the gamma-ray photons entering the material were determined. For each incident photon, the free path length $l$ was sampled from the exponential distribution

$$
l=-\ln (q) / \mu_{t},
$$

where $\mu_{t}$ is the total attenuation coefficient (sum of incoherent (Compton) attenuation and photoelectric absorption coefficients) for investigated sample. A fitted equation for attenuation coefficients, given in Eq. (3), was obtained for the energy range between 10 and $2000 \mathrm{keV}$ using the cross sections of photoelectric absorption and incoherent (Compton) scattering:

$$
\mu=\exp \left(p_{1}+p_{2} x+p_{3} x^{2}+p_{4} x^{3}+p_{5} x^{4}\right),
$$

where $p_{1}, p_{2}, p_{3}, p_{4}$ and $p_{5}$ are the parameters of photoelectric absorption and incoherent scattering for each attenuating material, and $x=\ln E(\mathrm{keV})$.

Coordinates of the interaction points of the gammaray photon entering the attenuator were determined, and checks were made whether this point was within the material. If it was found to be inside the material, then the type of photon interaction was sampled. If it was decided that the interaction was photoelectric, a new gammaray photon was sampled. If the interaction was Compton scattering, the cosine of the scattering polar angle was calculated using a rejection technique, reported by
Özmutlu [18], based on the free electron Klein-Nishina expression, and the coordinates of the next interaction point were determined using the direction cosines and sampled free path.

This procedure was continued until the photon was depleted of all of its energy or had left the medium. If the photon left the attenuator, it was checked whether the photon had reached the detector. In this way, the number of photons reaching the detector, called transmitted intensity $I$ of photons, was obtained. By plotting $\ln \left(I_{0} / I\right)$ versus $x$, the slope equal to linear attenuation coefficient was calculated.

Half-value layer (HVL) and tenth-value layer (TVL) were obtained using the following equations:

$$
\begin{aligned}
& \mathrm{HVL}=\ln 2 / \mu, \\
& \mathrm{TVL}=\ln 10 / \mu .
\end{aligned}
$$

The mean free path $(\mathrm{mfp})$ is given as:

$$
\operatorname{mfp}=1 / \mu \text {. }
$$

\section{Results and discussion}

Transmitted intensities of gamma-rays $I$ have been determined using a simple Monte Carlo code for calculation of linear attenuation coefficients of some phantom materials, that were previously used by Hill et al. for testing the water equivalence of these materials [16]. The simulation process was repeated for several thicknesses, up to approximately $6 \mathrm{mfp}$, and the linear attenuation coefficients of these materials were calculated by plotting $\ln \left(I_{0} / I\right)$ versus $x$. In the simulation, the phantom materials were irradiated by photons with the energies of 59.5, $80.9,140.5,356.5,661.6,1173.2$ and $1332.5 \mathrm{keV}$.

The calculated Monte Carlo linear attenuation coefficients versus incident photon energy are presented in Table II, as well as the coefficients measured by Hill et al. [16] and the coefficients calculated using the XCOM and EGSnrc [16]. The simulation results agree with the measured values and with the calculated EGSnrc and XCOM linear attenuation coefficients. The values derived from XCOM are greater than those derived from our Monte Carlo code. This result is consistent with those of Hill et al. [16] and Reniers et al. [19].

The discrepancy in the values of the calculated and the measured coefficients could be due to deviations from narrow beam geometry in the source-detector arrangements, as suggested by Medhat [14] and Gurler and Akar Tarim [5].

The values of our calculated linear attenuation coefficients for each solid phantom material are compared with the coefficients that were found for water at seven different gamma-ray energies (Fig. 1a-d). The results indicate that Perspex has a significant deviation in the linear attenuation coefficient values, while the RMI-457 solid water, plastic water, and RW3 solid phantoms have similar values. 
TABLE II

Linear attenuation coefficients of water and four solid phantom materials, calculated by Monte Carlo method (this study), using EGSnrc [16] and XCOM databases [20], and the measured values [16].

\begin{tabular}{|c|c|c|c|c|c|c|c|c|c|c|c|c|c|c|c|c|c|c|c|c|}
\hline \multirow[b]{3}{*}{$\begin{array}{c}\text { Energy } \\
{[\mathrm{keV}]}\end{array}$} & \multicolumn{20}{|c|}{ Linear attenuation coefficients $\left[\mathrm{cm}^{-1}\right]$} \\
\hline & \multicolumn{4}{|c|}{ Water } & \multicolumn{4}{|c|}{ RMI-457 } & \multicolumn{4}{|c|}{ Plastic water } & \multicolumn{4}{|c|}{ RW3 } & \multicolumn{4}{|c|}{ Perspex } \\
\hline & 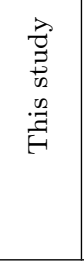 & 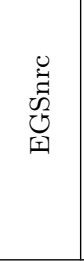 & $\begin{array}{l}\sum_{0} \\
0 \\
0 \\
x\end{array}$ & 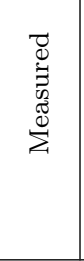 & $\begin{array}{l}\vec{\theta} \\
\vec{E} \\
\vec{B} \\
.0 \\
\vec{E} \\
\vec{H}\end{array}$ & 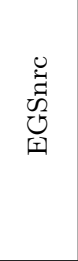 & $\begin{array}{l}\sum_{0} \\
0 \\
\bigcup_{x}\end{array}$ & 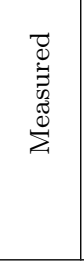 & 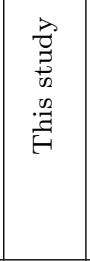 & 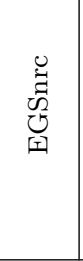 & $\begin{array}{l}\sum_{0} \\
0 \\
\bigcup_{x}\end{array}$ & 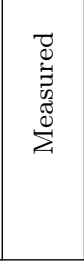 & 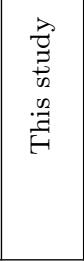 & 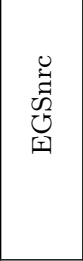 & $\begin{array}{l}\sum_{0} \\
0 \\
0 \\
x\end{array}$ & 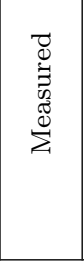 & 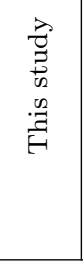 & 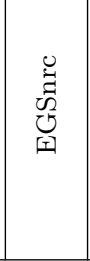 & $\begin{array}{l}\sum_{0} \\
0 \\
\circlearrowright \\
\searrow\end{array}$ & 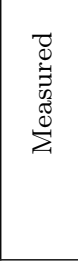 \\
\hline 59.5 & 0.192 & - & 0.207 & - & 0.193 & - & 0.209 & - & 0.226 & - & 0.239 & - & 0.188 & - & 0.204 & - & 0.211 & - & 0.230 & - \\
\hline 80.9 & 0.171 & - & 0.183 & - & 0.170 & - & 0.184 & - & 0.181 & - & 0.195 & - & 0.171 & - & 0.182 & - & 0.190 & - & 0.207 & - \\
\hline 140.5 & 0.146 & 0.151 & 0.154 & 0.148 & 0.148 & 0.151 & 0.154 & 0.151 & 0.150 & 0.152 & 0.156 & 0.151 & 0.148 & 0.153 & 0.155 & 0.149 & 0.168 & 0.170 & 0.177 & 0.166 \\
\hline 356.5 & 0.108 & - & 0.111 & - & 0.108 & - & 0.112 & - & 0.108 & - & 0.111 & - & 0.110 & - & 0.113 & - & 0.124 & - & 0.128 & - \\
\hline 661.6 & 0.084 & - & 0.086 & - & 0.084 & - & 0.086 & - & 0.084 & - & 0.086 & - & 0.085 & - & 0.087 & - & 0.096 & - & 0.099 & - \\
\hline 1173.2 & 0.064 & - & 0.065 & - & 0.064 & - & 0.066 & - & 0.064 & - & 0.065 & - & 0.064 & - & 0.066 & - & 0.073 & - & 0.075 & - \\
\hline 1332.5 & 0.060 & - & 0.061 & - & 0.060 & - & $|0.061|$ & - & $|0.059|$ & - & 0.061 & - & 0.060 & - & 0.062 & - & 0.069 & - & 0.070 & - \\
\hline
\end{tabular}

Comparison of HVL, TVL and mfp va- TABLE III lues of water and phantom materials.

\begin{tabular}{|c|c|c|c|c|}
\hline & Energy [keV] & HVL [cm] & TVL [cm] & $\mathrm{mfp}[\mathrm{cm}]$ \\
\hline \multirow{7}{*}{ Water } & 59.5 & 3.61 & 11.99 & 5.21 \\
\hline & 80.9 & 4.05 & 13.46 & 5.85 \\
\hline & 140.5 & 4.75 & 15.77 & 6.85 \\
\hline & 356.5 & 6.42 & 21.32 & 9.26 \\
\hline & 661.6 & 8.25 & 27.41 & 11.90 \\
\hline & 1173.2 & 10.83 & 35.98 & 15.62 \\
\hline & 1332.5 & 11.55 & 38.37 & 16.67 \\
\hline \multirow{7}{*}{ RMI-457 } & 59.5 & 3.59 & 11.93 & 5.18 \\
\hline & 80.9 & 4.08 & 13.54 & 5.88 \\
\hline & 140.5 & 4.68 & 15.56 & 6.76 \\
\hline & 356.5 & 6.42 & 21.32 & 9.26 \\
\hline & 661.6 & 8.25 & 27.41 & 11.90 \\
\hline & 1173.2 & 10.83 & 35.98 & 15.62 \\
\hline & 1332.5 & 11.55 & 38.37 & 16.67 \\
\hline \multirow{7}{*}{$\begin{array}{c}\text { Plastic } \\
\text { water }\end{array}$} & 59.5 & 3.07 & 10.19 & 4.42 \\
\hline & 80.9 & 3.83 & 12.72 & 5.52 \\
\hline & 140.5 & 4.62 & 15.35 & 6.67 \\
\hline & 356.5 & 6.42 & 21.32 & 9.26 \\
\hline & 661.6 & 8.25 & 27.41 & 11.90 \\
\hline & 1173.2 & 10.83 & 35.98 & 15.62 \\
\hline & 1332.5 & 11.75 & 39.03 & 16.95 \\
\hline \multirow{7}{*}{ RW3 } & 59.5 & 3.69 & 12.25 & 5.32 \\
\hline & 80.9 & 4.05 & 13.46 & 5.85 \\
\hline & 140.5 & 4.68 & 15.56 & 6.76 \\
\hline & 356.5 & 6.30 & 20.93 & 9.09 \\
\hline & 661.6 & 8.16 & 27.09 & 11.76 \\
\hline & 1173.2 & 10.83 & 35.98 & 15.62 \\
\hline & 1332.5 & 11.55 & 38.37 & 16.67 \\
\hline \multirow{7}{*}{ Perspex } & 59.5 & 3.28 & 10.91 & 4.74 \\
\hline & 80.9 & 3.65 & 12.12 & 5.26 \\
\hline & 140.5 & 4.13 & 13.71 & 5.95 \\
\hline & 356.5 & 5.59 & 18.57 & 8.06 \\
\hline & 661.6 & 7.22 & 23.98 & 10.42 \\
\hline & 1173.2 & 9.50 & 31.54 & 13.70 \\
\hline & 1332.5 & 10.05 & 33.37 & 14.49 \\
\hline
\end{tabular}

Additionally, it can be seen from Fig. 1a-d that the linear attenuation coefficient of each phantom material decreases sharply in the low energy range, and then becomes almost constant in the medium energy range. This would imply that if we increase the energy of the incident photons we would obtain smaller attenuation, and therefore more significant penetration of the rays in the media $[21,22]$.
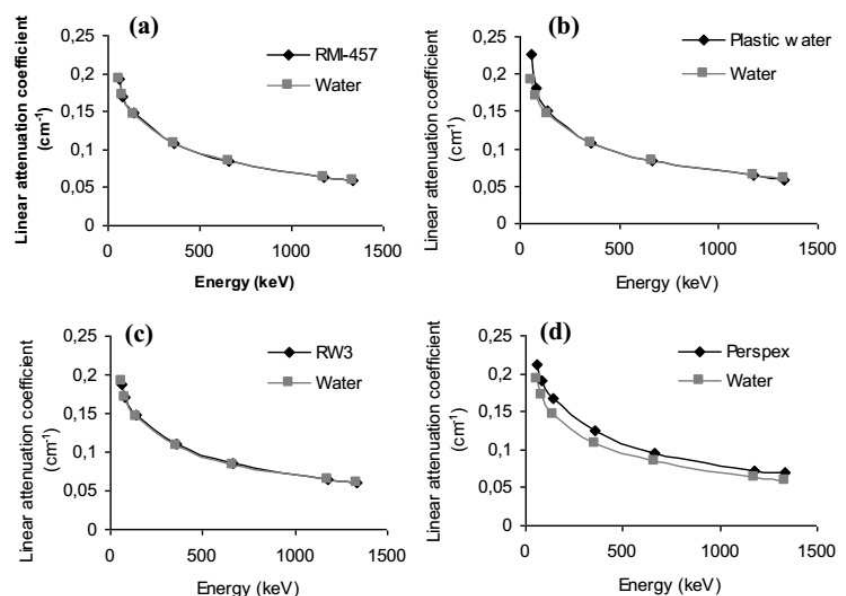

Fig. 1. Comparison between the linear attenuation coefficients of water and the linear attenuation coefficients of (a) RMI-457, (b) plastic water, (c) RW3 and (d) Perspex, to test the water equivalency of these solid phantom materials.

The half-value layers, tenth-value layers and mean free paths of water and four phantom materials for different radiation energies have been obtained and are presented in Table III. The lower the values of half-value layers and tenth-value layers, the better is the radiation shielding material in terms of the thickness requirements. 


\section{Conclusions}

In this project, a simple Monte Carlo code was implemented as an alternative to the experimental approach, in order to determine the linear attenuation coefficients. Comparison of Monte Carlo calculated values with the values obtained by three other methods shows that this method can be used for various attenuating materials and energy values. Additionally, the water equivalency of four phantom materials was evaluated and the results were found to be consistent with those of Hill et al. [16]. Furthermore, we have observed that the linear attenuation coefficients of phantom materials decrease only slightly in the medium energy range (356.5, 661.6, 1173.2 and $1332.5 \mathrm{keV})$. This result agrees with the conclusions reported by Gurler and Akar Tarim [5] and Medhat [14]. Having observed this agreement, three other shielding parameters, namely the half-value layer, tenth-value layer, and mean free path, were also calculated using the simulation results.

\section{Acknowledgments}

We would like to express our sincere gratitude to Prof. Dr Emin N. Ozmutlu for his fitting program and guidance.

\section{References}

[1] N. Singh, K.J. Singh, K. Singh, H. Singh, Radiat. Meas. 41, 84 (2006).

[2] E. Yılmaz, H. Baltas, E. Kırıs, İ. Ustabas, U. Cevik, A.M. El-Khayatt, Ann. Nucl. Energy 38, 2204 (2011).

[3] I.I. Bashter, Ann. Nucl. Energy 24, 1389 (1997).

[4] I. Akkurt, C. Basyigit, S. Kilincarslan, B. Mavi, A. Akkurt, Cement Concrete Compos. 28, 153 (2006).

[5] O. Gurler, U. Akar Tarim, J. Radioanalytical Nucl. Chem. 293, 397 (2012).
[6] M. Kurudirek, İ. Türkmen, Y. Özdemir, Radiat. Phys. Chem. 78, 751 (2009).

[7] İ. Türkmen, Y. Özdemir, M. Kurudirek, F. Demir, Ö. Simsek, R. Demirboğa, Ann. Nucl. Energy 35 , 1937 (2008)

[8] N. Damla, U. Cevik, A.I. Kobya, A. Celik, N. Celik, R.V. Grieken, J. Hazardous Mater. 176, 644 (2010).

[9] C. Bootjomchai, J. Laopaiboon, C. Yenchai, R. Laopaiboon, Radiat. Phys. Chem. 81, 785 (2012).

[10] H. Singh, K. Singh, L. Gerward, K. Singh, H.S. Sahota, R. Nathuram, Nucl. Instruments Meth. Phys. Res. B 207, 257 (2003).

[11] M.N. Alam, M.M.H. Miah, M.I. Chowdhury, M. Kamal, S. Ghose, R. Rahman, Appl. Radiat. Isotopes 54, 973 (2001).

[12] D.V. Raje, L.M. Chaudhari, Bulgarian J. Phys. 37, 158 (2010).

[13] A.F. Baytaş, S. Akbal, Radiat. Meas. 35, 17 (2002).

[14] M.E. Medhat, Ann. Nucl. Energy 36, 849 (2009).

[15] I. Akkurt, H. Akyıldırım, B. Mavi, S. Kilincarslan, C. Basyigit, Ann. Nucl. Energy 37, 910 (2010).

[16] R.F. Hill, S. Brown, C. Baldock, Radiat. Meas. 43, 1258 (2008).

[17] Z. Moravek, L. Bogner, Zeitschrift Medizinische Physik 19, 174 (2009).

[18] E.N. Özmutlu, Appl. Radiat. Isotopes 43, 713 (1992).

[19] B. Reniers, F. Verhaegen, S. Vynckier, Phys. Med. Biol. 49, 1569 (2004).

[20] M.J. Berger, J.H. Hubbell, S.M. Seltzer, J. Chang, J.S. Coursey, R. Sukumar, D.S. Zucker, K. Olsen, www.nist.gov/pml/xcom-photon-cross-sectionsdatabase, 2010.

[21] M.L. Cortes, F. Cristancho, Study of gamma backscattering in a multielemental medium, 2007.

[22] I. Akkurt, Ann. Nucl. En. 36, 1702 (2009). 